\title{
DENSITY OF STATES AND INDIRECT EXCHANGE IN METALLIC SYSTEMS
}

\author{
A. Hernando ${ }^{a, b}$, J.M. Rojo ${ }^{a}$, J.C. Gómez SAL ${ }^{c}$ AND J.M. BarandiaráN ${ }^{d}$ \\ ${ }^{a}$ Departamento de Física de Materiales, Universidad Complutense, Madrid, Spain \\ ${ }^{b}$ Instituto de Magnetismo Aplicado, UCM, CSIC, RENFE, Las Rozas \\ P.O. Box 155, Madrid 28230, Spain \\ 'Departamento de Materia Condensada, Universidad de Cantabria, Santander, Spain \\ ${ }^{d}$ Departamento de Electricidad y Electrónica, Universidad del Pais Vasco, Bilbao, Spain
}

(Received July 26, 1996)

\begin{abstract}
The relation between the density of states at the Fermi level and the sign of the indirect coupling in metallic systems is revisited in the framework of a simple model recently outlined by the authors. It is shown that high density of states tends to favor ferromagnetic structures. Some examples which support this idea are indicated. Finally we develop a phenomenological explanation for the antiferromagnetic $\rightarrow$ ferromagnetic transition induced by heating equiatomic FeRh. The transition is analysed in terms of the increase in the density of states produced by the giant volume expansion associated with the ferromagnetic phase.
\end{abstract}

PACS numbers: 75.25.tz, 75.30.Et, 75.50.Bb

\section{Introduction}

As is well known, direct exchange, although conceptually simple, does not seem to be the underlying mechanism which orients localised spins in magnetic materials [1]. Indirect exchange, on the contrary, appears to be prominent but the mechanisms which contribute to it still need a better understanding. Only in. rather simple cases, such as rare earth magnetism, is indirect exchange straightforwardly explained [2] in terms of a conduction-electron mediated Ruderman-KittelKasuya-Yoshida (RKKY) interaction. Even in such conspicuous materials as $3 d$ metals are, an accurate description of their magnetism is still lacking. Although RKKY is believed to be the responsible interaction, the symmetry of the electrons actually contributing to the exchange between the localised magnetic ions is open to discussion and according to an extended view [3] one is even compelled to distribute the $3 d$ electrons in two ranks: localised and itinerant.

The recent surge of a number of new magnetic materials has revived the practical interest in these basic questions. Magnetic multilayers, granular systems, 
amorphous metals and series of new compounds are being currently investigated both for their industrial interest and their magnetic properties. In fact, some of these properties have turned out to be rather unexpected and, curiously enough, their study is throwing light in a number of long standing problems which had arised in connection with simpler, more traditional materials.

In response to the wealth of new phenomenology a number of theoretical analyses have been undertaken in order to elucidate the various intricate contributions to the magnetism of these systems. Many of these theoretical investigations are quite involved [4], as required by the subtle changes which are intrinsic to the accurate description of magnetic properties. Too often, alas, the underlying physical aspects are somehow obscured by the formalism and a first-order critical inventory of the most relevant contributions would benefit the understanding.

A particularly rewarding way of looking at indirect exchange in metals is by considering the perturbation introduced by a magnetic field to the conduction-electron Hamiltonian [5]. First- and higher-order perturbative terms can be neatly separated, each of them making a distinctive physical contribution to the magnetism of the system. For example, the first-order term always results in a Zener-like positive (ferromagnetic) component to the exchange. In this review we intend to use this approach to discuss the antiferro- (AF) and ferromagnetic (FM) states of a number of systems, particularly when they transit from one to the other state.

\section{Conduction band polarization}

The response of an electron conduction band to a periodic external magnetic drive

$$
\boldsymbol{H}(\boldsymbol{x})=V^{-1} \sum_{q} h_{q} \exp (\mathrm{i} \boldsymbol{q} \cdot \boldsymbol{x})
$$

where $V$ is the volume, can be described in terms of the so-called susceptibility $\chi$, which may be Fourier expanded to

$$
\chi=\sum_{q} \chi_{q} \exp (\mathrm{i} q \cdot x) .
$$

It is easy to see that the total magnetic energy $E$ can then be written as $E_{0}+E_{1}$ where

and

$$
E_{0}=\chi_{0} h_{0}^{2}
$$

$$
E_{1}=\sum_{q} \chi_{q} h_{q}^{2}
$$

$h_{q}$ being the Fourier components of the magnetic field. A straightforward first-order perturbation calculation, in which $\boldsymbol{H}(\boldsymbol{x})$ is taken as the perturbation agent leads to the so-called (using Van Vleck's terminology [6]) static component

$$
\chi_{0}=-\mu_{\mathrm{B}}^{2} N\left(E_{\mathrm{F}}\right)
$$

in terms of $N\left(E_{\mathrm{F}}\right)$ - the density of states at the Fermi level. The calculation of the other susceptibility components, named polarization terms, has been performed only for free electrons. The result is [5]

$$
\chi_{q}(q \neq 0)=\left(3 N \mu_{\mathrm{B}}^{2} / 4 E_{\mathrm{F}}\right)\left[1+\left(4 k_{\mathrm{F}}^{2}-q^{2}\right) \log \left|\left(2 k_{\mathrm{F}}+q\right) /\left(2 k_{\mathrm{F}}-q\right)\right| / 4 k_{\mathrm{F}} q\right] .
$$


The above expressions for $\chi_{0}$ and $\chi_{q}$ are based on a model of independent electrons. Exchange and correlation among the electrons in the band can be introduced through a parameter $v$ resulting in an enhancement of the independent spin susceptibility [7]

$$
\chi_{0}^{\text {eff }}=\chi_{0} /\left(1-v \chi_{0}\right) \quad \text { and } \quad \chi_{q}^{\text {eff }}=\chi_{\mathrm{q}} /\left(1-v \chi_{q}\right) .
$$

The difference between the energy of the ferro- and antiferromagnetic ordering of spins is

$$
E_{\mathrm{FM}}-E_{\mathrm{AF}}=-\mu_{\mathrm{B}}^{2} N\left(E_{\mathrm{F}}\right)+\sum_{q} \chi_{q}{ }^{\mathrm{FM}} h_{q}^{2}-\sum_{q} \chi_{q}{ }^{\mathrm{AF}} h_{q}^{2}
$$

where ${ }^{\mathrm{FM}} h_{q}^{2}$ designs the $q$-component of the magnetic field in a ferromagnetic array of spins and ${ }^{\mathrm{AF}} h_{q}^{2}$ - the corresponding value in an antiferromagnetic one.

\subsection{Free electrons}

We have recently evaluated [8] the energy balance (6) for a two-dimensional periodic array of magnetic moments, coupled by layers of non-magnetic material, the latter supplying the conduction electrons, assumed to be free. The polarization contribution (second and third terms in the right hand side of Eq. (7)) is found to be always positive, whereas the static one, equal to $-3 \mu_{\mathrm{B}}^{2} n / 2 E_{\mathrm{F}}(\mathrm{n}=$ density of conduction electrons), is negative, the sign of the coupling being determined by a competition between both contributions. A well known property [6] of free electrons is the following. The value of (4) exactly cancels the dc value around which (5) oscillates, i.e. the value of (5) for large $q$. Therefore, for an electron gas the oscillation of (5) leads to an oscillation from FM to AM of the total coupling.

\subsection{Bloch electrons}

For electrons interacting with a periodic potential, the expression (4) is still valid. This is not the case for the polarization components (5); in fact, a calculation of $\chi_{q}$ for $q \neq 0$ has not been performed. Nevertheless, there are reasons [8] to support the assumption that the polarization components do not change much when going from free to Bloch electrons. Under this assumption the coupling sign becomes controlled by the value of $N\left(E_{\mathrm{F}}\right)$, a large value of the latter favouring the FM case. It is worth emphasising that this is a property of non-interacting electrons; the introduction of corelation corrections, as in (6), only leads to enhanced parameters of the oscillation.

\section{Rare earth compounds and FM $\rightarrow$ AM transitions}

In real systems (far from a free electron gas) $N\left(E_{\mathrm{F}}\right)$ is then expected to control the coupling sign. In order to have an experimental check of these predictions, it would be desirable to have a direct determination of $N\left(E_{\mathrm{F}}\right)$ across an isomorphic series of materials in which transitions from AF to FM coupling were known to take place as a function of composition. Ideally, a technique should be chosen which would directly probe $N\left(E_{\mathrm{F}}\right)$. Recently [9] we have reported thermopower measurements across the $\mathrm{GdNi}_{1-x} \mathrm{Cu}_{x}$ and $\mathrm{NdNi}_{1-x} \mathrm{Cu}_{x}$ series and derived from 
these data the evolution with the concentration $x$ of $N\left(E_{\mathrm{F}}\right)$. Basing on this, we show that switching from $F M$ to AF can be better understood in terms of variation of the density of states rather than of the RKKY oscillation period (as a function of distance between ions) and, therefore, provide new evidence in support of the proposal of Ref. [8].

Information about $N\left(E_{\mathrm{F}}\right)$ can be obtained from techniques other than thermopower. The effective magnetic moment is a simple magnetic quantity which can be obtained from magnetic measurements above $T_{\mathrm{c}}$ and application of the high temperature Curie-Weiss law; the value so obtained is in general different from the theoretical rare earth free ion moment. The difference $\Delta \mu$ between the effective and theoretical values can be simply related to the $N\left(E_{\mathrm{F}}\right)$. Both our own measurements in $\mathrm{GdNi}_{1-x} \mathrm{Cu}_{x}$ and those of Skolozdra et al. [10] in stannides reveal a significant correlation between $\Delta \mu$ and the paramagnetic Curie temperature $\Theta$. This is tantamount to saying that there is correlation between $N\left(E_{\mathbf{F}}\right)$ and the sign of the coupling between the magnetic moments. In particular, when $N\left(E_{\mathrm{F}}\right)$ is reduced, the coupling becomes AF.

\section{The role of $N\left(E_{\mathrm{F}}\right)$ on partially delocalised systems}

It is possible to extend the considerations established above, on the indirect exchange of localised electrons (on the magnetic ions), to the more general case of exchange between partially localised electrons (PLE) coupled through exchange carrier electrons (ECE). Moreover, the ECE can be the PLE themselves (direct exchange) or electrons from a different orbital or band (indirect exchange).

With great generality the exchange mechanism consists in the effect of the partially localized moments (PLM) on the ECE. Any PLM, of strength $\mu$ located at $r=0$, produces a magnetization of the ECE which consists in a static term, $m_{0}$, parallel to the magnetic moment, plus a spatial polarization $m(r)$ opposite to the magnetic moment at any $r \neq 0$. Another PLM located at $r$ should be then subjected to a molecular field, $h(r)$, proportional to $m_{0}-m(r)$. The condition of ferromagnetism, $m_{0}>m(r)$, implies $\chi_{0} h_{0}>\sum \chi_{q} h_{q} \mathrm{e}^{\mathrm{i} q r}$ for any $r$. Since $\chi_{0}$ is proportional to $N\left(E_{\mathrm{F}}\right)$, the condition of ferromagnetism becomes $\chi_{0} h_{0} \gg 1$, which, by taking into account that $h_{0}$ is proportional to the exchange coupling between the PLM and the ECE, coincides with the Stoner condition of ferromagnetism established for the particular case of itinerant electrons, i.e. a sample to be ferromagnetic needs a high value of the product of the exchange and the density of states.

Let us now illustrate with some examples the relevant role played by $N\left(E_{\mathrm{F}}\right)$ as concerns the dependence of the magnetic order parameters on the interatomic distances. On the basis of a straightforward application of RKKY ideas, one could suppose that lattice expansions resulting in increased interionic distances would lead to a decrease in the exchange interactions between the PLE; however, a great majority of experimental observations indicate that ferromagnetism is reinforced by increasing interatomic distances. This behaviour supports the idea that $N\left(E_{\mathrm{F}}\right)$, which is expected to increase with volume expansions, is much more sensitive to the interatomic distances than the exchange term.

An example is the enhancement of both magnetic moment and Curie tem- 
perature induced by hydrogenation or nitrogenation in $3 d-4 f$ intermetallic compound used as material for permanent magnets [11]. These processes generate an increase in the lattice constant and therefore an increase in $N\left(E_{\mathrm{F}}\right)$. Another indication of the sensitivity of $N\left(E_{\mathrm{F}}\right)$ to the lattice constant is the usual positive sign of the volume magnetostriction. There are two examples which neatly illustrate the relationship existing between lattice expansion and reinforcement of the ferromagnetism. These examples are (i) the room temperature stress induced ferromagnetism in amorphous $\left(\mathrm{Fe}_{0.92} \mathrm{Zr}_{0.08}\right)_{96} \mathrm{~B}_{4}$ and (ii) the $1 \%$ volume expansion exhibited by bcc ordered $\mathrm{Fe}_{50} \mathrm{Rh}_{50}$ during the antiferromagnetic to ferromagnetic transformation, which undergoes at $400 \mathrm{~K}$.

$$
\text { 4.1. }\left(F_{0.92} \mathrm{Zr}_{0.08}\right)_{96} \mathrm{~B}_{4}
$$

Recently Barandiarán et al. [12] have shown that a large dependence of the Curie temperature on the pressure, similar to that exhibited by FeZr metallic glasses, is also characteristic of $\mathrm{Fe} \mathrm{ZrB}(\mathrm{Cu})$ ones. As the boron concentration increases, the Invar character decreases, but the weak itinerant ferromagnetism evolves toward a strong ferromagnetic behaviour. The effect of boron seems to be an increase in $N\left(E_{\mathrm{F}}\right)$. Some magnetic and Mössbauer experiments have pointed out that both Curie temperature and hyperfine field increase linearly with the applied tensile stress. The average hyperfine field increases at a typical rate of $4 \mathrm{~T} / \mathrm{GPa}$. Figure 1 shows the influence of the applied tensile stress on the thermal dependence of the magnetization for the sample $\mathrm{Fe}_{87} \mathrm{Cu}_{1} \mathrm{Zr}_{6} \mathrm{~B}_{6}$ measured in a field of $2500 \mathrm{~A} \mathrm{~m}^{-1}$. A large shift of the Curie point is evident.

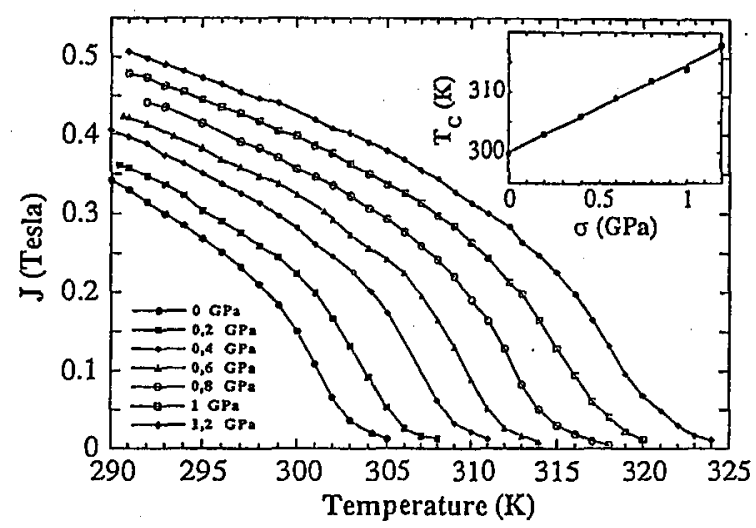

Fig. 1. Thermal evolution of the polarization, $J$, at $2.5 \mathrm{kA} / \mathrm{m}$, as a function of the applied tensile stress for a $\mathrm{Fe}_{87} \mathrm{Cu}_{1} \mathrm{Zr}_{6} \mathrm{~B}_{6}$ metallic glass. The inset illustrates the linear change in Curie temperature induced by the stress.

The Curie temperature of the sample with composition $\left(\mathrm{Fe}_{0.92} \mathrm{Zr}_{0.08}\right)_{96} \mathrm{~B}_{4}$ lies below but very close to room temperature. The strong effect of the tensile stress can be directly observed in the magnetization curve shown in Fig. 2. The appearance of the hysteresis loop and the broadening of the Mössbauer spectra at $291 \mathrm{~K}$ occurs at an applied tensile stress of $0.6 \mathrm{GPa}$. 

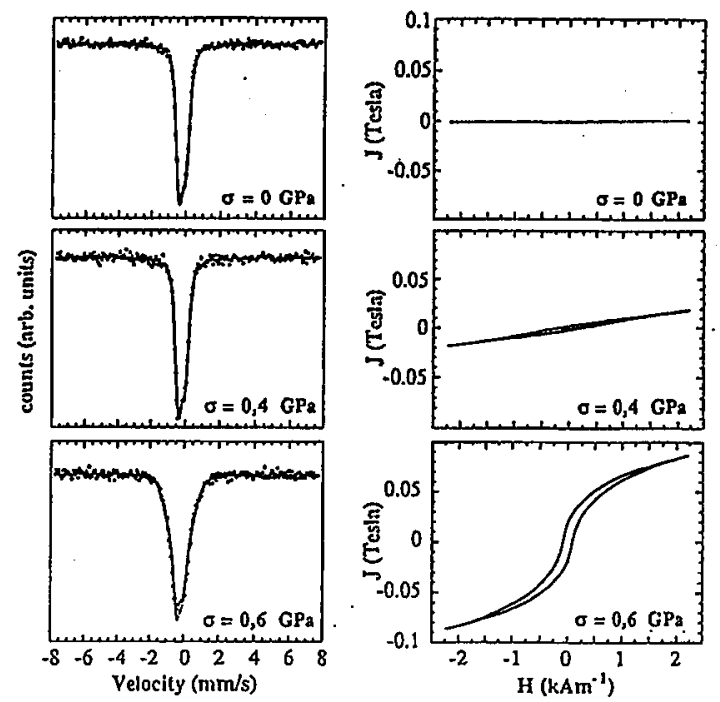

Fig. 2. Onset of ferromagnetism observed on the Mössbauer spectra and the magnetization curve, for a tensile stress of $0.6 \mathrm{GPa}$ applied at room temperature, on the paramagnetic alloy $\left(\mathrm{Fe}_{0.92} \mathrm{Zr}_{0.08}\right)_{96} \mathrm{~B}_{4}$.

According to the Stoner criterium, the increase in the Curie temperature, and therefore in the ferromagnetic character, of these samples when subjected to tensile stress could be associated with either an increase in the exchange or with an increase in the density of states. In general, one expects a decrease in the wave function overlapping with increasing interatomic distances and thereby in the exchange coupling. However, the volume expansion should increase $N\left(E_{F}\right)$ through the shortening of the distances between energy levels. Hence the most logical explanation of the stress induced ferromagnetism, at room temperature in $\left(\mathrm{Fe}_{0.92} \mathrm{Zr}_{0.08}\right)_{96} \mathrm{~B}_{4}$ amorphous alloys, is the increase in $N\left(E_{\mathrm{F}}\right)$ produced by the stress. This argument which can be also invoked to account for the general positive character of the volume magnetostriction will be shown to be useful in order to understand the next example.

\section{2. $A F \rightarrow F M$ transition in $F e_{50} R h_{50}$}

Those intermetallic compounds which undergo either $\mathrm{FM} \rightarrow \mathrm{AF}$ or $\mathrm{AF} \rightarrow \mathrm{FM}$ transition exhibit an anomalous volume expansion in the FM phase. This is the case of compounds as $\mathrm{Hf}_{1-x} \mathrm{Ta}_{x} \mathrm{Fe}_{1.98}, \mathrm{Nd}_{x} \mathrm{~Tb}_{1-x} \mathrm{Mn}_{2} \mathrm{Ge}_{2}, \mathrm{CeFe}_{2}$ or $\mathrm{Eu}_{x} \mathrm{Sr}_{1-x} \mathrm{~S}$. It turns out that the increase in $N\left(E_{\mathrm{F}}\right)$ associated to such expansion make energetically favourable the FM configurations in systems in which AF and FM configurations have similar energy. In particular, the transition from the low temperature phase, $\mathrm{AF}$, to the high temperature $\mathrm{FM}$ configuration which takes place in the $\mathrm{CsCl}$ like ordered $\mathrm{Fe}_{50} \mathrm{Rh}_{50}$ has been thoroughly studied $[13,14]$. The experimental characteristics of the transformation can be summarised as follows. (a) In the low temperature AF configuration the magnetic moment of the Fe atoms 
is $3 \mu_{\mathrm{B}}$ with no appreciable $\mathrm{Rh}$ moments. In the FM phase the Fe moment remains unchanged whereas the Rh moment rises to $1 \mu_{\mathrm{B}}$. (b) At the transition temperature, during heating, the thermal expansion exhibits a drastic rise producing $1 \%$ volume expansion, even though the crystallographic structure remains the same. (c) The AF $\rightarrow$ FM transition is associated with a large increase in entropy [15], corresponding to the induction of the Rh magnetic moments. (d) The transition temperature decreases with increasing applied magnetic field.

Let us now analyse the AF $\rightarrow$ FM transformation in connection with the volume expansion observed during the magnetic transition. The free energy of the AF, $F_{\mathrm{AF}}$, and FM, $F_{\mathrm{FM}}$, configurations should be equal at the transition temperature, $T_{\mathrm{t}}(H)$, which also depends on the applied field $H$. The condition $F_{\mathrm{AF}}=F_{\mathrm{FM}}$ at $H=0$ becomes

$$
U_{0}+E_{\mathrm{AF}}=U_{0}+E_{\mathrm{FM}}(\Delta v / v)-T_{\mathrm{t}} \Delta S+(1 / 2) \kappa(\Delta v / v)^{2},
$$

where $U_{0}$ is the internal energy associated with the lattice, $E_{\mathrm{AF}}$ and $E_{\mathrm{FM}}$ are the $\mathrm{AF}$ and FM magnetic energies as defined in (7), $\Delta S$ is the increase in entropy observed experimentally [15] which is of the order of $12 \mathrm{~mJ} /(\mathrm{g} \mathrm{K})$ or $8 \times 10^{4} \mathrm{~J} /\left(\mathrm{m}^{3} \mathrm{~K}\right)$ approximately, $\kappa$ - the compressibility, $2 \times 10^{11} \mathrm{~J} / \mathrm{m}^{3}$, and $\Delta v / v$ - the volume expansion of about $1 \%$. Notice that we have remarked in the right hand side of (8) the dependence of $E_{\mathrm{FM}}$ on $\Delta v / v$, which takes place through the dependence of $\chi_{0}$ on $N\left(E_{\mathrm{F}}\right)$ and the influence of $\Delta v / v$ on $N\left(E_{\mathrm{F}}\right)$.

As $T_{\mathrm{t}}$ is experimentally found to be about $400 \mathrm{~K}, E_{\mathrm{AF}}$ lies, according to (8), approximately $2.2 \times 10^{7} \mathrm{~J} / \mathrm{m}^{3}$ below $E_{\mathrm{FM}}(\dot{\mathrm{C} v} / \mathrm{v})$. Therefore the difference between the minimum of the magnetic energy $E_{\mathrm{AF}}$ and $E_{\mathrm{FM}}(0)$ is larger than $2.2 \times 10^{7} \mathrm{~J} / \mathrm{m}^{3}$ which corresponds to $0.3 \mathrm{eV}$ per atom. Therefore, the transition is driven by the increase in entropy and with the help of the volume expansion which decreases $E_{\mathrm{FM}}$ through the increase in $N\left(E_{\mathrm{F}}\right)$ and consequently in $\chi_{0}$.

Relation (8) has to be modified when an external magnetic field is applied. Under the action of the field two new energy terms must be considered. The terms $(-1 / 2) \mu_{0} \chi_{\mathrm{FM}} H^{2}$ and $(-1 / 2) \mu_{0} \chi_{\mathrm{FM}} H^{2}$ should be included to the left and right hand side of (8), respectively. $\chi_{\mathrm{AF}}$ and $\chi_{\mathrm{FM}}$ are the macroscopic susceptibility in the AF and FM configurations, respectively. Neglecting the thermal dependence of the susceptibility it is possible to approximate the field dependence of $T_{t}$, through the modified relation (8), as

$$
T_{\mathrm{t}}(H)=T_{\mathrm{t}}(0)-(1 / 2) \mu_{0}(\Delta \chi / \Delta S) H^{2} .
$$

Experimental results [16] show that the difference $\dot{C} \chi=\chi_{\mathrm{FM}}-\chi_{\mathrm{AF}}$ is about 5 for $H=5 \mathrm{~T}$, hence $T_{\mathrm{t}}$ decreases with the square of $H$ at a rate of $14 \mathrm{KT}^{-1}$. This result is in excellent agreement with the experimental determination of $T_{\mathrm{t}}(H)$ carried out by Ibarra and Algarabel [17].

\section{Conclusions}

The model recently developed by the authors [8] leads to the conclusion that the indirect exchange has two components of opposite sign, (i) ferromagnetic coupling which is very sensitive to the states placed nearby the Fermi level and (ii) antiferromagnetic polarization rather sensitive to all the states of the band. 
According to this model $N\left(E_{\mathrm{F}}\right)$ is the key parameter governing the total exchange coupling sign. When $N\left(E_{\mathrm{F}}\right)$ is larger than the expected for a parabolic band, from the average effective mass of the band, the coupling is ferromagnetic, whereas it becomes antiferromagnetic for the opposite case.

We have shown some examples which point out the remarkable importance of $N\left(E_{\mathrm{F}}\right)$ in determining the magnetic configuration of the ground state under different conditions. In particular it was shown that the magnetovolume effect reflects the influence of $N\left(E_{\mathrm{F}}\right)$ on the magnetic structures. In all the cases the ferromagnetism is associated with a volume expansion. In our model it can be understood as a consequence of the subsequent increase in $N\left(E_{\mathrm{F}}\right)$ which turns out to favour the magnetic energy of the FM configuration through the increase in $\chi_{0}$.

\section{Acknowledgments}

The authors wish to thank their coworkers J. Fontcuberta, R. Ibarra and R. Yavari for helpful discussions and continuous support. This work has been partially supported by the spanish CICyT, through projects Mat 95-1042 and Mat 95-0273.

\section{References}

[1] It is not always realised that the Bethe-Slater curve, in which direct exchange seems to be prominent in $3 d$ ferromagnetic metals, is only an educated guess. Most of the calculations carried out thereafter result in a much too low magnitude or even a wrong sign for the direct exchange coupling (R. Stuart, W. Marshall, Phys. Rev. 120, $353(1960))$.

[2] See for instance D.C. Mattis, in: The Theory of Magnetism I, Springer-Verlag, Berlin 1981 p. 229.

[3] M. Beath Sterns, Physics Today 31, 34 (1978) and references therein.

[4] A flavour of the sophistication attained in the rapidly evolving field of multilayers can be obtained in the recent review by Y. Yafet, in: Magnetic Multilayers, Eds. L.H. Bennett, R.E. Watson, World Scientific, Singapore 1994, p. 19.

[5] For a review see C. Kittel, in: Solid State Physics, Vol. 22, Eds. F. Seitz, D. Turnbull, H. Ehrenreich, Academic, New York 1968, p. 1.

[6] J.H. Van Vleck, Rev. Mod. Phys. 34, 681 (1962).

[7] B. Giovanni, M. Peter, J.R. Schrieffer, Phys. Rev. Lett. 12, 736 (1966).

[8] A. Hernando, J.M. Rojo, J.C. Gómez-Sal, J.M. Novo, J. Appl. Phys. 79, 4815 (1996).

[9] A. Hernando, J. Fontcuberta, J.C. Gómez Sal, J.M. Rojo, submitted to Phys. Rev. B.

[10] R. Skolozdra, R. Szymczak, H. Szymczak, L. Romaka, M. Baran, J. Phys. Chem. Solids 57, 357. (1996).

[11] J.M.D. Coey, D.H. Ryanand, Yu Boliang, J. Appl. Phys. 55, 1800 (1994).

[12] J.M. Barandiarán, P. Gorría, I. Orue, M.L. Fernandez Gubieda, F. Plazaola, A. Hernando, Phys. Rev. B 54, 3026 (1996).

[13] V.L. Moruzzi, P.M. Marcus, Phys. Rev. B 46, 2864 (1992).

[14] R.C. Wayne, Phys. Rev. 170, 523 (1968).

[15] J.S. Kouvel, J. Appl. Phys. 37, 1257 (1966).

[16] M. Fallot, R. Hocart, Rev. Sci. 77, 498 (1939).

[17] M.R. Ibarra, P.A. Algarabel, Phys. Rev. B 50, 4196 (1994). 